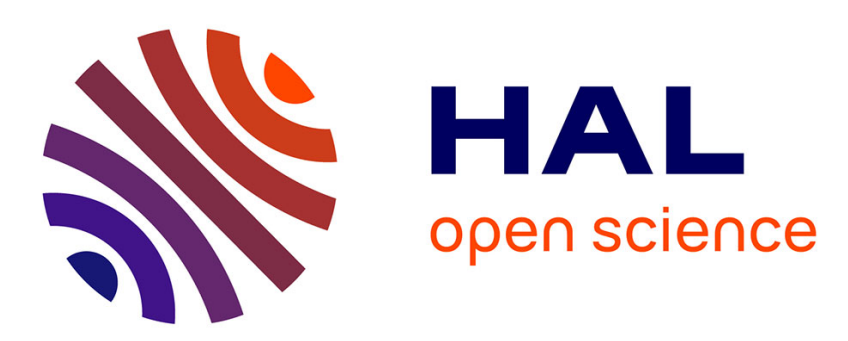

\title{
Histoplasmosis Caused by Histoplasma capsulatum var. duboisii : A Comprehensive Review of Cases From 1993 to 2019
}

\author{
M Develoux, F M Amona, C Hennequin
}

\section{- To cite this version:}

M Develoux, F M Amona, C Hennequin. Histoplasmosis Caused by Histoplasma capsulatum var. duboisii : A Comprehensive Review of Cases From 1993 to 2019. Clinical Infectious Diseases, 2021, 73 (3), pp.e543-e549. 10.1093/cid/ciaa1304 . hal-03365987

\section{HAL Id: hal-03365987 \\ https: / hal.sorbonne-universite.fr/hal-03365987}

Submitted on 5 Oct 2021

HAL is a multi-disciplinary open access archive for the deposit and dissemination of scientific research documents, whether they are published or not. The documents may come from teaching and research institutions in France or abroad, or from public or private research centers.
L'archive ouverte pluridisciplinaire HAL, est destinée au dépôt et à la diffusion de documents scientifiques de niveau recherche, publiés ou non, émanant des établissements d'enseignement et de recherche français ou étrangers, des laboratoires publics ou privés. 
Histoplasmosis caused by Histoplasma capsulatum var. duboisii: a comprehensive review of the cases from 1993 to 2019

M. Develoux ${ }^{1}$, F. M. Amona ${ }^{2,3,4}$, C. Hennequin ${ }^{5}$

1. Assistance Publique-Hôpitaux de Paris, Hôpital St Antoine, Service de Parasitologie-Mycologie, F75012 Paris, France

2. Faculty of Health Sciences, Marien Ngouabi University, BP: 69 Brazzaville, Republic of Congo

3. Laboratory of Parasitology-Mycology, Edith Lucie Bongo Ondimba General Hospital, Oyo, Republic of Congo

4. Research Center and Study of Infectious and Tropical Pathologies, Oyo, Republic of Congo

5. Sorbonne Université, Inserm, Centre de Recherche Saint-Antoine, CRSA, AP-HP, Hôpital SaintAntoine, Service de Parasitologie-Mycologie, F-75012 Paris, France

Corresponding author: Pr. C. Hennequin, Service de Parasitologie-Mycologie, Hôpital St Antoine,

34 rue Crozatier, F-75012, Paris, France

Phone: +33 149283412

Fax: +33149282472

Email: christophe.hennequin@upmc.fr

Alternate corresponding author: Dr Michel Develoux

Service de Parasitologie-Mycologie, Hôpital St Antoine,

34 rue Crozatier, F-75012, Paris, France

Phone: +33 149283413

Fax: +33149282472

Email: micheldeveloux@yahoo.fr 
40-word summary

A large review of cases of Histoplasma duboisii histoplamosis highlights epidemiologic features and clinical characteristics, notably in HIV-infected patients. Diagnostic tools are suboptimal. The overall mortality rate reaches $23.4 \%$. Efficient antifungal drugs should be widely available in African endemic countries. 


\begin{abstract}
Background: Histoplasmosis caused by Histoplasma capsulatum var. duboisii (Hcd) is a rare, but probably under-estimated, endemic infection described in intertropical Africa. Its epidemiology remains unclear, the distinction with Histoplasma capsulatum var. capsulatum distributed worldwide (including in Africa) is complex and there is no consensus on the therapeutic management.
\end{abstract}

Methods: By use of a comprehensive search on different internet databases, we collected case reports of Hcd infection published from 1993 to 2019. Epidemiological and clinical charts and therapeutic strategies were analyzed.

Results: We found 94 well-documented cases of $\mathrm{Hcd}$ infection. $30.1 \%$ of the patients were under 18 . Symptoms occurred in some patients several decades after leaving the endemic area. Cutaneous/subcutaneous lesions, bone infection and lymphadenopathies, isolated or combined, were the most frequent presentations. The HIV co-infection rate was at $20.8 \%$ with fever, lymphadenopathies and absence of bone infection being the differentiating elements from HIV-negative patients. The rate of disseminated forms (60.6\% in our review) significantly increased as compared to studies published before 1993 but without correlation with HIV infection. The global mortality rate was at $23.4 \%$ by the end of follow-up. The outcome was not correlated with the antifungal drug prescribed nor with the HIV serologic status but with the initiation of an antifungal therapy.

Conclusion: $\mathrm{Hcd}$ histoplasmosis is a severe fungal infection which precise mode of acquisition remains to be determined. There is a need for affordable and more specific diagnostic tools. Itraconazole and amphotericin B are the best therapeutic alternatives and should be available in all low-income countries of the endemic area.

Keywords: African histoplasmosis; Histoplasma capsulatum var. duboisii; epidemiology; HIV; diagnostic; treatment 


\section{Introduction}

H. capsulatum, a dimorphic fungus responsible for human histoplasmosis, encompasses two varieties, namely $H$. capsulatum var. duboisii $(H c d)$ and $H$. capsulatum var. capsulatum $(H c c)$ [1]. Dubois et al first described $\mathrm{Hcd}$ in 1952 in the Belgian Congo, today the Republic of Congo [2]. A year later Vanbreusegem used the parasitic and saprophytic characters to distinguish between the two varieties [3]. Furthermore, epidemiological and clinical characteristics have been described for each of the infections due to the 2 varieties $[4,5]$. Notably since $H c d$ infection has only been reported from Africa, the disease is commonly referred as "African histoplasmosis". However, both varieties has been described in Africa and recent phylogenetic analyses support the monophyly of $H$. capsulatum on this continent, with the existence of a unique African clade including both $H c c$ and $H c d$ strains $[6,7]$. Thus, these results and other suggest that the classification, based only on the geographic origin of the acquisition, is obsolete [8].

In Africa, $H c c$ and $H c d$ are endemic in at least 32 countries, but $H c d$ infection reports overpass those on Hcc infection [5, 9]. Nevertheless, $H c d$ histoplasmosis is considered as a rare fungal infection, occurring in Sub-Saharan African countries and Madagascar, but the disease is not included in the group of the Neglected Tropical Diseases [9]. Since the first description of the disease, several papers over the years have reviewed its main characteristics [10-12]. In 1993, Carme et al recorded 237 Hcd histoplasmosis cases without detailing their characteristics [13]. The aim of the present review is to stand out the possible new aspects regarding this infection by the mean of a large review of the literature from 1993. Notably, the role of HIV co-infection on the clinical presentation and outcome is analyzed. 


\section{Methods}

For the present study, we searched for new cases or series published after the publication of Carme et al in 1993 [13]. This was done using the PubMed database with the limitations of date 1993-2019 and with the keywords: "African histoplasmosis", "histoplasmosis AND Africa", "Histoplasma duboisii", "Histoplasma capsulatum var duboisii". We included all publications whatever was the language, notably French and Portuguese ones. In addition, we retrieved from other internet sources (Google scholar notably) some cases published in non-referenced journals.

We only considered well-documented cases and so excluded cases described in conference abstract books or presented in case-series with limited details. For each case, we analyzed the demographic information, the clinical presentation, results of radiological and microbiological investigations, the mode of diagnosis and the therapeutic management. We focused particularly on cases occurring in HIVinfected patients, as they emerged as an at-risk population in our analysis.

\section{Statistics}

Comparisons were done by the mean of the Fisher's exact or Chi-square test for qualitative variables and the Student t-test for quantitative variables using the Prism (v7.0a) software. 


\section{Results}

We found a total of 147 cases with a clear demonstration of yeasts supporting the diagnosis of $\mathrm{Hcd}$ infection (Supplementary Table S1). Seventeen and 36 cases were included in the two most important case-series reported from Togo and Republic of Congo, respectively [14, 15]. Those cases were excluded from the clinical and therapeutic parts of the analysis due to insufficient description of the cases. Thus, 94 well-documented cases were reviewed to depict at the best the main characteristics of Hcd infections.

\section{Epidemiology}

The sex-ratio $(\mathrm{M} / \mathrm{F})$ was at 2.4 and the mean age at age at $30.7 \pm 17.5$ ranging from 13 months to 68 years. Children under 18 represented $30.1 \%$ of the cases. Patients originated from Central and Western Africa in 141 out of 147 cases (Figure 1).

\section{Clinical presentation}

The main clinical presentations are summarized in Table 1 and illustrated in Figure 2. Cutaneous and subcutaneous lesions, osteoarticular infections and lymphadenopathies were the most frequent, isolated or combined, and seen in $69(74.2 \%), 44(46.8 \%)$ and 39 cases $(41.9 \%)$, respectively. Twenty-seven patients were febrile at the time of diagnosis. In 37 cases (39.4\%), the infection presented as a single lesion, mainly localized either to the skin $(n=13)$, or to a bone $(n=9)$ but 57 patients $(60.6 \%)$ had disseminated infection affecting at least 2 non-contiguous body-sites. Compared to a 1972 report from Drouhet, the frequency of disseminated form increased from $16.4 \%$ of the cases (19 out of 116) [12] to $60.6 \%$ in our review (Fischer's exact test; $\mathrm{p}<0.0001$ ). Disseminated forms were not significantly more frequent in HIV-positive (81.8\% versus 62.1\%) (Fisher's exact test p=0.11) (Table 2).

The cutaneous lesions could be either unique $(n=31)$ or multiple $(n=38)$. They involved preferentially the limbs $(n=68)$, the trunk $(n=54)$, and the face $(n=59)$. Their appearance was very diverse and sometimes polymorph for a given patient. Cutaneous and subcutaneous nodules, maculo-papules, abscesses were the most common with the multiple umbilicated papulo-nodules simulating molluscum contagiosum observed in 21 patients in the case of dissemination. 
Lymphadenopathies were either superficial $(n=37)$ and/or deep-seated $(n=6)$. Superficial lymphadenopathies could be firm or indolent, fixed or mobile sometimes painful and localized with equal frequency in the inguinal $(n=23)$, axillary $(n=24)$ and cervical chains $(n=24)$. A fistula to the skin was seen in 10 cases.

Osseous infection was frequently multifocal ( 25 out of 44 cases). Infections of the spine ( $n=14)$, the thorax ( $n=15)$, bones of the upper $(n=18)$ and lowers $(n=18)$ limbs were the most common. In 11 cases spinal infection mimicked a Pott's disease [16-18]. Lesions were always osteolytic without reconstruction. In 4 cases, they were revealed or complicated by a pathologic fracture [19-22].

Lungs infections was clinically or radiologically suspected in 10 cases but mycologically confirmed in only 3 cases. Adrenal gland infection and nasal or buccal mucosal infection was reported in 2 and 4 cases, respectively.

Abdominal infection was diagnosed in 8 patients (8.5\%) either in the form of gastro-intestinal tract infection (gastric or duodenal lesions $(\mathrm{n}=2)$, acute colitis $(\mathrm{n}=1)$ ) or as intra-abdominal infection such as mass $(n=2)$, abscess $(n=1)$, peritoneal nodules or peritonitis $(n=2)$.

Gathering the data of cases reports and of the more important case-series [14, 15], 25 out of $120(20.8 \%)$ tested patients were found HIV-positive. Fever was most common in HIV+ patients (59.1 vs 22.4\%; Fisher's exact test $\mathrm{p}=0.003)$ and we found a lower frequency in bone infection 18.2 vs $58.6 \%(\mathrm{p}=0.002$; Fisher's exact test) and a trend toward a higher frequency of lymph nodes infection 63.6 vs $38.6 \%$ (Fisher's exact test; $\mathrm{p}=0.08$ ) in those patients compared to HIV- patients.

\section{Laboratory diagnosis}

Among the 94 observations reviewed, the diagnosis relied on direct examination of different fluids or skin scrapings, or histopathological examination of a biopsy specimen in 50 and 74 cases, respectively. A confirmation of the diagnosis using either culture or PCR was only obtained in 26 and 7 cases, respectively. Among the 9 patients tested for serum antibodies, 3 were found positive. We did not find any case mentioning Histoplasma antigen detection neither B-D glucan assay. A single case of Hcd disseminated infection in an HIV infected woman reported with a positive galactomannan (GM) serum 
test, with a slow decrease in titer following antifungal treatment [23]. GM test was mentioned in another case but the assay remained negative in an immunocompetent woman with pulmonary and cutaneous lesions [24].

\section{Treatment}

The treatment regimen was specified for 90 out of 94 observations analyzed. Eight patients did not receive any treatment either because of a rapidly fatal outcome $(n=5)$, because they denied treatment $(n=1)$ or because of spontaneous healing or absence of relapse following surgery alone $(n=2)$. Thus, 82 patients received antifungal $\operatorname{drug}(\mathrm{s})$, either alone $(\mathrm{n}=59)$ or combined with surgery (drainage, debridement of necrotic tissues, osseous curettage) $(n=23)$. First-line therapy mainly relied on iv amphotericin B $(n=28)$ or one of its lipid formulations $(n=5)$, itraconazole $(n=27)$, fluconazole $(n=8)$ and ketoconazole ( $\mathrm{n}=13$ ), the latter being no longer recommended for systemic therapy (Table 3 ). In 7 cases, amphotericin B toxicity (renal failure, general symptoms) led to the discontinuation or a switch for another drug. The safety profile of itraconazole was overall good with only 2 cases of adverse events mentioned in 45 patients treated with this drug. Two patients with bone infection were treated with terbinafine, either as first-line or as maintenance therapy following iv amphotericin $\mathrm{B}$, leading to remission in both cases with a follow-up of 5 and 8 months, respectively [21, 25].

By the end of the first-line treatment, remission, failure and relapse were notified in $50(53.2 \%), 6(6.4 \%)$ and $9(9.6 \%)$ cases, respectively. We failed to demonstrate any significant difference in the success rate according to the antifungal drugs, but the mortality rate was significantly higher for patients that did not receive any antifungal drug as compared to those treated with antifungal(s): $75 \%$ vs $13.4 \%$ ( $\mathrm{p}=0.0004$ Chi-square).

By the end of follow-up, 22 (23.4\%) patients died but the final outcome was not specified for 18 cases and only 17 had a follow-up exceeding one year. We found a trend toward a better prognosis for localized infection compared to disseminated form (Fisher's exact test; $\mathrm{p}=0.0685$ ), which was not the case for the HIV serologic status which did not influence the outcome $(\mathrm{p}=0.76)$. 


\section{Discussion}

While not include in the official list of the WHO, Hcd histoplasmosis can be considered a neglected tropical disease. From the first report in 1952, nearly $400 \mathrm{Hcd}$ cases have been reported but the cases series are few so that the epidemiologic and clinical characteristics and the optimal therapeutic management remain incompletely known. In this review, we analyzed retrospectively 94 welldocumented cases allowing us to point out some of those characteristics.

As described in the past $[10,12]$, the majority of documented cases arose from Western and Central Africa, between latitudes $15^{\circ}$ North and $10^{\circ}$ South (Figure 1). However, a recruitment bias is likely as most of the cases are recorded from countries having long-standing medical structures able to diagnose the disease. More recently, cases have been reported from other countries such as Togo or GuineaBissau $[14,26]$, suggesting that the endemic area could be wider. However, given the coexistence of $H c c$ and $H c d$ in Africa, the use of the results of ecological studies (detection of the fungus in the soil or of guano specimens) or of intradermal tests of histoplasmin to specify the endemic area should be done with caution.

The hypothesis that the prevalence of the disease is underestimated is also supported by the fact that 28 cases out of 94 (29.8\%) have been diagnosed in countries outside Africa, mainly in Europe. The rural or urban origin of the patients was rarely specified in the literature, but people living in the bush or the forest, with outdoor activities, seemed to be the most affected [13, 27]. In a Togolese series, the majority of patients came from rural areas ( 15 out of 17 cases) just as in the Congolese series (10 out of 14 cases). Most had a low socio-economic level [14, 15].

The pathophysiology of $\mathrm{Hcd}$ infection remains largely unknown, notably its mode of acquisition. Inhalation of fungal spores is a possible route of infection. During the investigation of a particular focus of Hcd infection in the Democratic Republic of the Congo, four interviewed patients acknowledged collecting guano from caves to fertilize gardens [15]. However, only 3 out of the 94 cases we reviewed were diagnosed with a documented pulmonary infection [23, 28, 29]. A breach in the skin barrier is another possible mode of acquisition with several cases supporting this hypothesis: a man developed an 
ulcerated tumor at the needle prick of acupuncture after mud-baths [30], and another had superinfection of cicatricial lesions following tribal scarification rituals [31]. Similarly, the infection has been sometimes described after a cutaneous traumatism with initial symptoms at the site of the trauma [19, 32, 33]. In some cases, an intestinal penetration could have been the portal of entry as cases with isolated digestive involvement have been reported $[31,34,35]$.

Our review does not allow a precise estimation of the incubation time of $\mathrm{Hcd}$ infection. Cutaneous signs at the level of a local trauma have been reported 3 weeks to 2 months later $[19,32]$. In contrast, HIVnegative expatriates developed symptoms years (a minimum of 2) or decades after leaving the endemic area [31, 35-39]. So, whatever the date of leaving the endemic area is, the diagnosis of Hcd infection should not be ruled out before appropriate investigation.

Analysis of clinical charts highlights the very pleiomorphic presentation of the disease, so that the disease should be more commonly suspected. However, the most striking result is the increase in the incidence of disseminated infection over the 25 years of our review. The HIV co-infection has been previously suggested to explain this phenomenon [38]. However, we failed to demonstrate a significative difference in the incidence of disseminated form between HIV+ and HIV-patients. So, one can assume that this phenomenon could also be due to the more frequent use of high definition imaging, allowing the detection of lesion(s) that would have been previously ignored. In their review, Loulergue et al noted that fever (58\% versus $15 \%)$ and the lack of lung infection ( $0 \%$ versus $75 \%)$ were more common in HIV-positive patients [38]. In addition, we were able to point out the lower frequency of bone infection and the higher frequency of lymphadenopathy in those patients.

Probably due to the limited laboratory diagnosis opportunities in low-income countries, 55\% of the cases were diagnosed only on direct examination or histopathological analysis of clinical specimen(s) making some diagnosis questionable as already mentioned [40]. This is illustrated by the unique autochthonous case of $\mathrm{Hcd}$ infection diagnosed outside of Africa [41]. This patient from Southern India denied any travel outside this region. He presented with multiple erythematous papules on the trunk and the arms. The authors presented histopathological findings compatible with Histoplasma yeast forms. Cultures grew positive for a fungus further identified as $H$. capsulatum. This case underlines the difficulty in the 
definitive diagnosis of $H c d$ infection. Indeed, $H c c$ being, like in Africa, endemic in India, the diagnosis of $H c d$ infection only relies on the size of yeasts observed on the biopsy specimen, which can be questionable in this case. Several reports support the use of PCR for the diagnosis of Hcd infection [30, 42] but it should be recalled that neither the PCR-based methods nor the morphology of the colonies of obtained in culture, irrespective of the phase obtained either the mycelial or the yeast phase, do allow the differentiation between $H c c$ and $H c d$. Thus, a careful examination to characterize the morphology of the yeasts in tissue or fluids is crucial for a definitive diagnosis [40]. Large budding yeasts of 7-15 $\mu \mathrm{m}$ in diameter, ovoid, with thick cell walls are characteristic features [4]. Chain formation in the shape of an hourglass or figure-eight, bud scar and cytoplasmic lipid inclusions also support the diagnosis, but while these features are quite easy to recognize on direct examination of fluids, pathologic examination of biopsy specimen can be more difficult to interpret. This warrants the development of new diagnostic tests reliable and easy to perform in low-income countries.

To the best of our knowledge, there is no specific guidelines or consensus statement for the therapeutic management of $\mathrm{Hcd}$ infection. By analogy with $\mathrm{Hcc}$ infection, and in accordance with the IDSA guidelines, some experts recommend the use of iv amphotericin B deoxycholate or its lipid formulations, or itraconazole, according to the severity of the disease [38, 43]. However, it should be kept in mind that the patient's financial resources and the availability of drugs in the considered country probably most often drove the therapeutic option. Amphotericin B is not licensed in or unavailable in $22(14.2 \%)$ and $42(27.1 \%)$ out of 155 countries, respectively [44] and the daily price of deoxycholate amphotericin B ranges from $<\$ 1$ to $\$ 171$. Considering the lack of significative difference in the outcome according to the drug used, itraconazole, thanks to its oral formulation and its safety profile, appears as a valuable alternative. Therapeutic drug monitoring is desirable notably in the case of co-administration of enzyme inductor drugs such as rifampicin, but not widely available in low-income countries. Fluconazole has the advantage of being well-tolerated but its bone penetration is lower than itraconazole [45].

The optimal duration of therapy could neither be defined. Relapses have been reported up to 2 years after an initial improvement [23]. Bone lesions should probably be treated with a minimum course of 6 months, which represents a major drawback in the limited-resource setting. For HIV-infected patients 
with $\mathrm{Hcd}$ infection, the duration of therapy should depend obviously on the recovery of an efficient immune response. Immune reconstitution inflammatory syndrome has been reported in two patients with $H c d$ infection [46, 47], but recent guidelines do not recommend to delay the initiation of antiretroviral therapy in the context of opportunistic histoplasmosis (due to Hcc) [48].

\section{Conclusions}

Our review suggests that the prevalence of $H c d$ infection is underestimated because cases can be easily missed notably in low-income countries. Indeed, the clinical presentation is very pleiomorphic so that the disease should be suspected more frequently. HIV co-infection is common (20\% of the cases) and substantially modifies this clinical presentation. Diagnosis mainly relies on the direct examination which requires specific laboratory materials and microscopist skills, that are not always available in those countries. There is an urgent need for reliable and easy-to-use diagnostic tools to confirm the diagnosis and distinguish between $\mathrm{Hcd}$ and $\mathrm{Hcc}$ infections, which are both endemic on the African continent. Finally, the review points out the absolute necessity for the availability at a reduced price of the antifungal drugs used to treat $H c d$ infection, mainly iv amphotericin B and itraconazole. 
Cutaneous/Subcutaneous lesion(s) ( $\mathrm{n}=69$ cases)

\begin{tabular}{lcccc}
\hline & Face & Trunk & Limbs & Others \\
\hline $\begin{array}{l}\text { Cutaneous/sub-cutaneous } \\
\text { nodule or mass }\end{array}$ & 24 & 23 & 28 & 1 \\
$\begin{array}{l}\text { Molluscum contagiosum-like } \\
\text { Maculo-papules }\end{array}$ & 13 & 13 & 10 & \\
Cold abscess & 9 & 8 & 10 & 1 \\
Others & 6 & 7 & 13 & 1 \\
\hline
\end{tabular}

Lymph node(s) ( $\mathrm{n}=39$ cases $)$

\begin{tabular}{lclc}
\hline & Superficial & & Deep-seated \\
\hline Inguinal & 23 & Abdominal & 3 \\
Cervical & 24 & Mediastinal & 3 \\
Axillary & 24 & Retro-peritoneal & 2 \\
\hline
\end{tabular}

Bone infection $(\mathrm{n}=44$ cases $)$

\begin{tabular}{llcc}
\hline & & Multifocal (n=24) & Unifocal (n=19) \\
\hline \multirow{2}{*}{ Axis } & Skull & 4 & 4 \\
& Spine and sacrum & 11 & 3 \\
\hline \multirow{2}{*}{ Bony thorax and pelvic } & Ribs and sternum & 5 & \\
& Scapula and clavicle & 7 & 3 \\
& Pelvic bones & 2 & 5 \\
\hline \multirow{2}{*}{ Upper limbs } & Humerus & 7 & 1 \\
& Ulna and radius & 3 & 2 \\
\hline \multirow{2}{*}{ Lower limbs } & Hand bones & 2 & 1 \\
& Femur & 5 & \multirow{2}{*}{ Tibia and fibula } \\
\hline
\end{tabular}

Table 1: Main clinical characteristics of 94 patients with $\mathrm{Hcd}$ infection. 


\begin{tabular}{|c|c|c|c|c|}
\hline & $\begin{array}{c}\text { All } \\
\mathrm{n}=\mathbf{9 3}^{1}\end{array}$ & $\begin{array}{l}\text { HIV- } \\
n=57\end{array}$ & $\begin{array}{c}\text { HIV+ } \\
n=22\end{array}$ & HIV+ vs HIV- \\
\hline Sex ratio & 2.44 & 2.56 & 2.14 & NS \\
\hline Mean age \pm SD & $30.9 \pm 17.4$ & $29.5 \pm 19.2$ & $34.9 \pm 10.4$ & NS \\
\hline $\begin{array}{l}\text { Localized/Disseminated } \\
\text { infection }\end{array}$ & $37 / 56$ & $22 / 35$ & $4 / 18$ & $\mathrm{NS}(\mathrm{p}=0.11)$ \\
\hline Fever & $26(27.9 \%)$ & $12(21 \%)$ & $13(59.1 \%)$ & $\mathrm{p}=0.0024$ \\
\hline $\begin{array}{l}\text { Cutaneous/sub-cutaneous } \\
\text { infection }\end{array}$ & $69(74.2 \%)$ & $42(80.8 \%)$ & $17(77.3 \%)$ & NS \\
\hline Lymph nodes & $39(41.9 \%)$ & $22(38.6 \%)$ & $14(63.6 \%)$ & NS $(p=0.0765)$ \\
\hline Osseous infection & $44(47.3 \%)$ & $33(57.9 \%)$ & $4(18.2 \%)$ & $\mathrm{p}=0.0022$ \\
\hline Miscellaneous & $20(21.5 \%)$ & $10^{2}(15.8 \%)$ & $8^{3}(36.4 \%)$ & NS \\
\hline Global mortality & $21(22.6 \%)$ & $14(24.6 \%)$ & $6(27.3 \%)$ & NS \\
\hline
\end{tabular}

Table 2: Comparison of clinical features of 94 patients with $\mathrm{Hcd}$ infection according to the HIV serologic status

NS: not significative

${ }^{1} \mathrm{HIV}$ serologic status was not specified for 14 patients

${ }^{2}$ Intra-abdominal infection ( $\left.n=3\right)$; Adrenal glands $(n=2)$, Lungs $(n=1)$, Digestive tract $(n=2)$, mucosal infection $(\mathrm{n}=2)$

${ }^{3}$ Bone marrow $(n=2)$, Digestive tract $(n=1)$, Intra-abdominal infection $(n=1)$, mucosal infection $(n=2)$, Lungs + digestive tract $(\mathrm{n}=1)$, Lungs + liver + spleen + bone marrow $(\mathrm{n}=1)$ 
